\title{
Preparation of an Amphiphilic RAFT agent and its application in organic phthalocyanine blue pigment dispersion
}

\author{
Lianbing Zhang ${ }^{1, a^{*}, \text { Rui Chen }}{ }^{2, b}$ and Chao Zhang ${ }^{3, c}$ \\ ${ }^{1}$ School of Textile and Garment, Hebei University of Science and Technology, Shijiazhuang, China \\ ${ }^{2}$ Hebei branch China nuclear power engineering co., Ltd, Shijiazhuang, China \\ ${ }^{3}$ Tianjin Yadong Chemical Co., Ltd, Tianjin, China \\ alianbingzhang@hotmail.com, bchenrui@ cnpe.cc, ${ }^{\mathrm{c}}$ magic-chao@qq.com
}

\begin{abstract}
Keywords: RAFT agent, dispersion, pigment, organic phthalocynine.
Abstract. An amphiphilic RAFT agent was synthesized, which could polymerize with monomers as Reversible Addition-Fragmentation Chain Transfer Polymerization route. The resulting RAFT agents were characterized by FT-IR and ${ }^{1} \mathrm{H}$ NMR. The dispersing ability of synthesized RAFT agent was evaluated. Organic phthalocyanine blue pigment was dispersed with the amphiphilic RAFT agents by vigorously stirring and ultrasonic processing. The properties (particle size, polydispersity index, stability) of pigment dispersions were measured. The results showed that prolonging ultrasonication time the particle size and PDI reduced first and increased slightly later.
\end{abstract}

\section{Introduction}

Pigment is one of the most colorants for textile. Compared to dyes, pigment has much more superiority because of higher light and wash fastness, suitable for all kinds of fibers and fabrics, shorter printing procedure. [1] However, because of their low polarity, organic pigments always existed in aggregation or coagulation powder. They were water-insoluble, hard to be wetted in aqueous media, and generally applied as aqueous dispersions. [2,3]

The dispersants play an important role in preparing pigment dispersion. They are amphiphilic agents that have hydrophilic and lipophilic segments. The dispersants can adsorb on the particle surface via anchoring segments (lipophilic), forming a layer around the particles which extend into the surrounding medium to provide entropic stabilization. [4] Meanwhile chains could prevent particle aggregations by steric and static repulsion.

RAFT (Reversible Addition-Fragmentation chain Transfer) method was a sophisticated form of controlled free radical polymerization, first introduced at CSIRO in 1998[5, 6], was one of the most convenient and versatile living radical polymerization techniques. This technology had prominent advantages such as the ability to prepare polymers with narrow molecular weight distribution, good compatibility with a wide range of monomers, facile experimental conditions that were similar to conventional radical polymerization. In RAFT polymerization, the key point was the RAFT agent that could react with monomers controlled.

In this work, an amphiphilic RAFT agent was synthesized. Its dispersing ability for organic phthalocyanine was evaluated.

\section{Experimental}

Reagents

Water was distilled and deionized before use. The organic phthalocyanine blue pigment, a gift from Hengshui Beilite Chemical Industry Co., Ltd., was used as supplied. Carbon disulfide, 1-dodecanethiol, sodium hydroxide, tetrabutyl ammonium bromide $\left(\left(\mathrm{C}_{4} \mathrm{H}_{9}\right)_{4} \mathrm{~N}^{+} \mathrm{Br}^{-}\right)$, hexane, acetone, 1, 4-dioxane $\left(\mathrm{C}_{4} \mathrm{H}_{8} \mathrm{O}_{2}\right)$, 2-bromopropanoic acid, from Sinopharm Chemical Reagent Co., Ltd were used as received.

Synthesis of RAFT agent

The amphiphilic RAFT agent 2-\{[(dodecylsulfanyl) carbonothioyl $]$ sulfanyl $\}$ propanoic acid was synthesized as follows. [7] 
Step I: A sodium hydroxide solution was added to a stirred mixture of dodecanethiol and water. Then solvents were added, the turbid mixture became clear and transparent.

Step II: The resulting clear solution was stirred and cooled to ambient temperature and treated with carbon disulfide to give a clear orange solution. Stirring for another $30 \mathrm{~min}, \alpha$-bromopropanoic acid was added and followed by sodium hydroxide solution, keeping the temperature below $10^{\circ} \mathrm{C}$.

Step III: The obtained orange solution was stirred at ambient temperature for $24 \mathrm{~h}$. Hydrochloric acid was added keeping the temperature below $10^{\circ} \mathrm{C}$, then yellow oil separated, stirring of the mixture until the oil solidified. The solid was collected by suction filtration, washed with cold water, and dried under reduced pressure. The crude sample was purified by recrystallizing to give bright yellow microcrystals.

\section{Pigment dispersion}

A solution containing, RAFT agent, water and sodium hydroxide was prepared in $50 \mathrm{~mL}$ beaker. To this solution P.B.15:3 was added, mixed and stirred, then dispersed with a JY98-3D Ultrasonic Pulverizer (Scientz company, China) at power $800 \mathrm{~W}$ (work model 1s/4s pulse on/off) for $10 \mathrm{~min}$.

\section{Measurements}

FT-IR spectra

FT-IR spectra were recorded on a NICOLET 6700 spectrometer (Thermo Fisher Scientific Inc., America) with a DTGS detector. The dried samples mixed with $\mathrm{KBr}$ were compressed into a disc for FT-IR scanning. The measurement was performed with 32 runs scanning and the resolution of $2 \mathrm{~cm}^{-1}$.

Particle size and its distribution

The particle size was measured at $25^{\circ} \mathrm{C}$ using a Malvern Instrument NANO-ZS 90 (Malvern Inc., England) at a fixed scattering angle of $90^{\circ}$. The dispersion was diluted with distilled water before measuring. Dynamic light scattering (DLS) measurements give a Z-average size and the polydispersity index (PDI), which provides the width of the particle size distribution.

Absorbency of pigment dispersion

The absorbencies of pigment dispersions were measured with a UV-5600 spectrophotometer (Shanghai metash instrument CO. Ltd, China) after diluting 1000 times with distilled water. Dilute pigment dispersion obeys the Lambert-Beer law. Absorbance values were measured at the maximum wave length $\left(\lambda_{\max }=620 \mathrm{~nm}\right)$ of the pigment dispersion.

\section{Results and Discussion}

\section{Preparation of RAFT agent}

In RAFT radical polymerization, a variety of thiocarbonythio compounds have been used, including dithiobenzoates, trithiocarbonates, xanthates, dithiocarbamates, and other compounds. In this research, RAFT agent with hydrophilic and lipophilic sections was synthesized according to the reaction mechanism of a carbodithionate salt with an alkylating agent, shown in scheme 1. [8]

In basic condition, thiol 1 (with $\mathrm{Z}$ group) ionized to anionic form 2 . Addition of carbon disulfide to 2 gave the thiocarbonylthio salt 3 . Without need for isolation, the trithiocarbonate can be made to interact with the alkylating agent, with the appropriate $\mathrm{R}$ group. The intermediate sodium salt 3 was not stable, easily oxidized in air, therefore the synthesis was processed in one pot, and the alkylating agent should react with 3 in situ.

The synthesized RAFT agent was characterized with FT-IR and ${ }^{1} \mathrm{H}-\mathrm{NMR}$ spectroscopy. Seen from Fig. 1, wave numbers of groups vibration were $v(\mathrm{O}-\mathrm{H}) 3432.73 \mathrm{~cm}^{-1}, v(\mathrm{C}-\mathrm{H}) 2955.02,2918.57$, $2851.25 \mathrm{~cm}^{-1}, v(\mathrm{C}=\mathrm{O}) 1706.35 \mathrm{~cm}^{-1}, v(\mathrm{C}-\mathrm{S}) 1209.20 \mathrm{~cm}^{-1}, \mathrm{v}(\mathrm{C}=\mathrm{S}) 1097.37 \mathrm{~cm}^{-1}$. Chemical shifts of hydrogen atoms were $\delta(\mathrm{ppm}) 10.89\left(\mathrm{br}, 1 \mathrm{H}, \mathrm{CO}_{2} \mathrm{H}\right), 4.87(\mathrm{q}, \mathrm{J}=7.4 \mathrm{~Hz}, 1 \mathrm{H}, \mathrm{SCH}), 3.36(\mathrm{t}, \mathrm{J}=7.4 \mathrm{~Hz}$, $2 \mathrm{H}, \mathrm{CH}_{2} \mathrm{~S}$ ), 1.70 (quint, $\mathrm{J}=7.4 \mathrm{~Hz}, 2 \mathrm{H}, \mathrm{CH}_{2} \mathrm{CH}_{2} \mathrm{~S}$ ), $1.63\left(\mathrm{~d}, \mathrm{~J}=7.4 \mathrm{~Hz}, 3 \mathrm{H}, \mathrm{SCHCH}_{3}\right.$ ), 1.39(sext, J $=7.44 \mathrm{~Hz}), 1.26\left(\mathrm{br}, 16 \mathrm{H}, \mathrm{CH}_{3} \mathrm{CH}_{2}\left(\mathrm{CH}_{2}\right)_{8}\right), 0.88\left(\mathrm{t}, \mathrm{J}=6.7 \mathrm{~Hz}, 3 \mathrm{H}, \mathrm{CH}_{3} \mathrm{CH}_{2}\right)$.

In preparation of the RAFT agent, its yields were affected by solvents, seen from table 3 . It was shown that yields of trithiocarbonate was increased with addition of tetrabutyl ammonium bromide and 
1, 4-dioxane. Dodecanethiol was hydrophobic, thus organic solvent facilitate it interact with carbon disulfide in water.

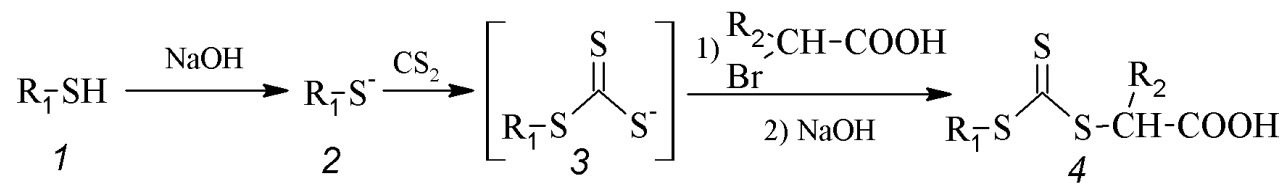

Scheme 1 Synthesis route of amphiphilic RAFT agent with carbon disulfide

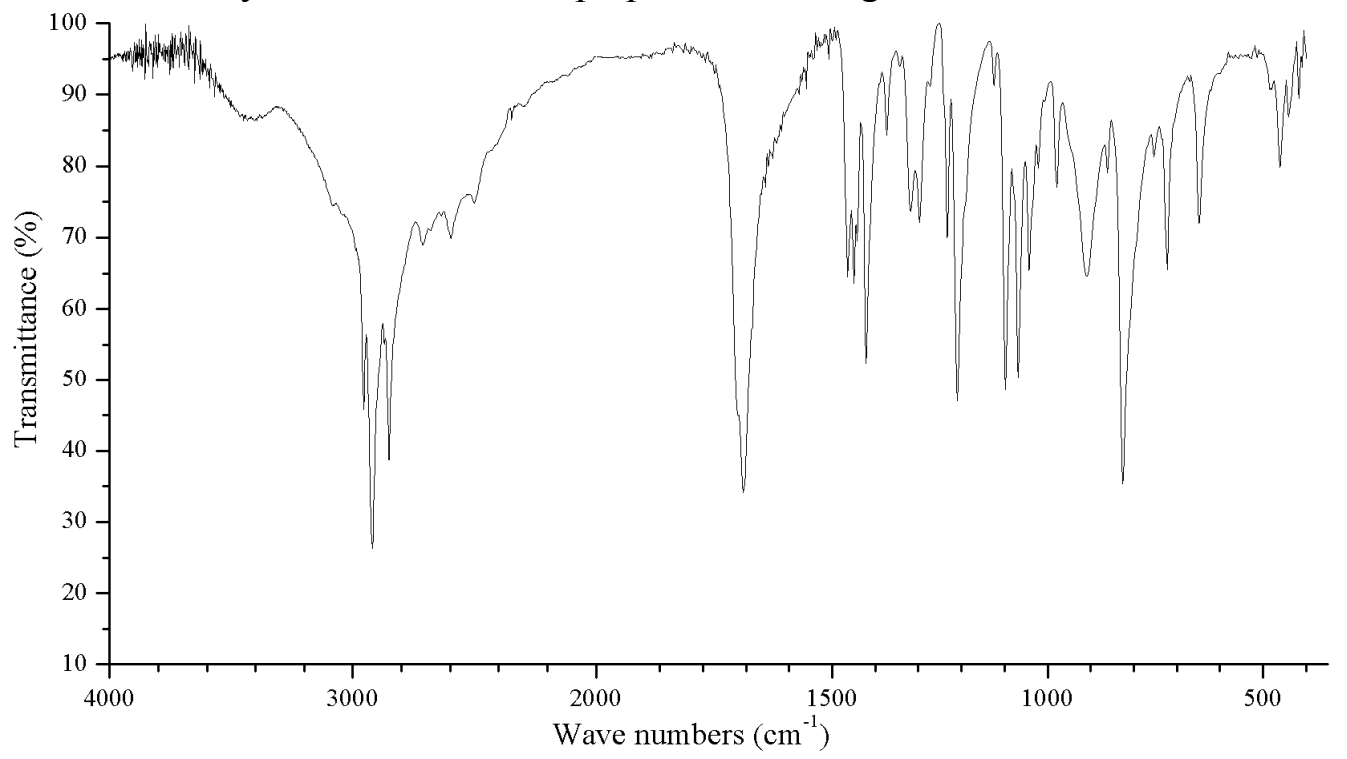

Fig. 1 FT-IR spectrum of synthesized RAFT agent

Table 1 Effects of solvents on yields of RAFT agents

\begin{tabular}{cc}
\hline Solvents & Yields $(\%)$ \\
\hline $\mathrm{H}_{2} \mathrm{O}$ & 28.8 \\
$\mathrm{H}_{2} \mathrm{O} /\left(\mathrm{C}_{4} \mathrm{H}_{9}\right)_{4} \mathrm{~N}^{+} \mathrm{Br}^{-} / \mathrm{C}_{4} \mathrm{H}_{8} \mathrm{O}_{2}$ & 55.7 \\
\hline
\end{tabular}

\section{Pigment dispersion}

Ultrasonication time

Pigment dispersing with hydrolyzed RAFT agents was evaluated. The alkyl hydrophobic region can interact with organic pigment. The hydrophilic carboxylic acid or its salt could provide the dispersion stability depending on steric and electrostatic repulsions.

Table 2 Effect of ultrosonication time on pigment dispersion

\begin{tabular}{ccc}
\hline Time $(\min )$ & Particle size $(\mathrm{nm})$ & PDI \\
\hline 1 & 210.7 & 0.375 \\
2 & 190.4 & 0.214 \\
3 & 186.8 & 0.230 \\
4 & 189.1 & 0.207 \\
5 & 185.9 & 0.217 \\
7 & 184.8 & 0.225 \\
9 & 182.4 & 0.211 \\
\hline
\end{tabular}

The pigment dispersions were produced by ultrasonication the mixture of pigment, hydrolyzed RAFT agents and water. In ultrasonication process, pigment aggregates were broken up to small particles because of ultrasonic cavitations. With longer time, the particle size of pigment was smaller, and the particle size distribution (PDI) decreased. After about $5 \mathrm{~min}$ ultrasonication, the particle size almost did not change, and PDI increased slightly. Except of deagglomeration, ultrasonic cavitation could generate heat. With longer time, heat generation made temperature rising, thus, Brownian movement of particles speeded up, smaller particles may interact with each other and result in particle agglomerations again, which made the particle size and PDI increased. Therefore, in ultrasonic 
dispersing procedure, the dispersion temperature must be kept as low as possible and too long process was not desirable.

\section{Amount of RAFT agents}

It revealed from table 3 that particle size of pigment dispersions reduced first and increased later, with increasing the amounts of RAFT agents. The variation of PDI was similar to that of particle size. The smaller particle size of pigment, the surface areas were larger, and also higher surface energy. Small particles tended to aggregate to larger ones to reduce surface energy. Therefore, there were much more amounts of dispersants required to adsorb on the surface of particles to prevent them from aggregating.

However, too many amounts of dispersants were not desirable. When the amount of RAFT agents was too high, a part of them would disperse in the water which could increase the viscosity of dispersion, thus led to lower dispersing efficiency. Moreover, RAFT agents dispersed in water might bridge two or more particles to form large aggregates. Thus, particle size, PDI and stability of pigment dispersion were poor.

Table 3 Effect of amounts of RAFT agents on pigment dispersions

\begin{tabular}{cccc}
\hline RAFT agent (wt \%) & Particle size $(\mathrm{nm})$ & PDI & Absorbance \\
\hline 3 & 175.2 & 0.234 & 0.124 \\
5 & 156.8 & 0.228 & 0.172 \\
7 & 154.8 & 0.219 & 0.184 \\
10 & 162.6 & 0.225 & 0.248 \\
20 & 169.7 & 0.292 & 0.196 \\
25 & 180.2 & 0.313 & 0.201 \\
\hline
\end{tabular}

\section{Conclusions}

An amphiphilic RAFT agent was synthesized according to the reaction mechanism of a carbodithionate salt with an alkylating agent. It was characterized by FT-IR and ${ }^{1} \mathrm{H}-\mathrm{NMR}$. Organic phthalocyanine blue pigment was dispersed with it by vigorously stirring and ultrasonic processing. It was shown that the particle size and PDI have relationship with ultrasonication time and the amounts of RAFT agents. With prolongation of ultrasonication time the particle size and PDI reduced first and increased slightly later. When the amounts of RAFT agents were too low or high, the particle size and PDI were larger, and stability was poor.

\section{Acknowledgement}

This work was supported by Project of Hebei Education Department (Z2015130) and Hebei University of Science and Technology Platform of Advanced Textiles Materials and Manufacture Technology.

\section{References}

[1] K. J. Fang, S. H. Wang, C. X. Wang, A. L. Tian, J. Appl. Polym. Sci. 107 (2008) 2949.

[2] S. H. Fu, K. J. Fang, J. Appl. Polym. Sci. 105 (2007) 317.

[3] Steiert N., Landfester K., Macromol. Mater. Eng. 292 (2007) 1111.

[4] Calbo L. J., Handbook of Coatings Additives, Marcel Dekker, New York, 1992.

[5] Chiefari J., Chong Y. K., Ercole F., Krstina J., Jeffery J., Le T. P. T., Mayadunne R. T. A., Meijs G.

F., Moad C. L., Moad G., Rizzardo E., Thang S. H., Macromolecules 31(1998) 5559.

[6] Moad G., Rizzardo E., Thang S. H. , Aust. J. Chem. 58 (2005) 379.

[7] Rong R., You Y., Tao W., J. Appl. Polym. Sci. 105 (2007) 398.

[8] Mayadunne R. A., Moad G., Rizzardo E., Tetrahedron. Lett. 43 (2002) 6811. 